九州大学学術情報リポジトリ

Kyushu University Institutional Repository

\title{
INFORMATION GAMES
}

Kai, Yu

Department of Home Life Scinece, Fukuoka Women's University

https://doi.org/10.5109/13336

出版情報: Bulletin of informatics and cybernetics. 20 (1/2), pp.55-70, 1982-03. Research Association of Statistical Sciences

バージョン :

権利関係 : 


\title{
INFORMATION GAMES
}

\author{
By \\ Yu KA $\mathbf{A I}^{*}$ \\ (Received November 24, 1981)
}

\begin{abstract}
In a game each player holds a strategy based upon the actions that he can actually take. The quality and the quantity of the feasible actions are determined by his ability or power in the game. Generally every player has no information on the others' powers, so it is one of the most important problems for them to know the powers. We treat such unknown games, played periodically, from the viewpoint of information theory. We discuss both non-cooperative and cooperative games and prove some sufficient conditions under which a player can get the full information on the other players' powers from sequential observations on the states. In two person games we show also optimal strategies with respect to the information.
\end{abstract}

\section{Introduction.}

The main problems of the game theory established by von Neumann and Morgenstern [9] are to find some solutions for the competitions of players' interests, and to show optimal strategies for the solutions. In the theory it is generally assumed that every player knows the game structure such as the sets of all feasible actions of the players and the payoff system according to the actions. However, the assumptions are not always satisfied in practical games. In complex games the skill and the experiences determine principally the issues of the games. For example, the skill and the experience in chess or 'go' provide the set of the feasible actions, and those in athletic games provide the force of every feasible action, which affects the payoff derived from the actions. Such abilities of a player are not usually known to the other players, thus the game structures are not always clear to the players. If the structure is unknown, each player must play cauciously not to suffer a serious loss and should make a point of getting the information on the others. Therefore in the present paper we discuss the unknown games, treat the information as the principle subject, and study how much information the players can gain.

We may mention that the powers and the strategies of the players decides the issues of the games. The strategy is the way to choose actions, which effects the issue directly. The power is practical abilities to play the game such as forces, proficiencies

\footnotetext{
* Department of Home Life Science, Fukuoka Women's University, Fukuoka 813, Japan.
} 
and feasibilities of actions, that is, the power shows the qualities and the quantities of the actions, and the powers provide the game structure. Thus the player, who wants to map out an optimal strategy, should make efforts to get the information on the others' powers as much and as soon as possible. We suppose, of course, every player knows his own power exactly. The more a player gets the information, the more he gains advantages over the others. We show an example of two-person zero sum game. The players are denoted by $P-1$ and $P-2$, whose action spaces are $A$ and $B$ respectively. Let $r(a, b), a \in A, b \in B$, be a payoff function, the value of which is paid to $P-1$ by $P-2$. If $P-1$ gets the information that the feasible actions of $P-2$ are restricted to a subset $B^{\prime}$ of $B$, he can expect more reward according to the following inequality ;

$$
\max _{a \in A} \min _{b^{\prime} \in B^{\prime}} r\left(a, b^{\prime}\right) \geqq \max _{a \in A} \min _{b \in B} r(a, b)
$$

If the payoff depends on the proficiency in the actions and each player does not know the one of the opponent, he will not able to estimate the value of $r(a, b)$. Thus if a player gets enough information on the opponent's proficiency, he can choose more effective actions.

On the other hand the information on the strategies is also valuable, because it brings every player great benefit to know the ways that the others choose their actions. Since a strategy of each player is determined according to his power, it is impossible to estimate the other's strategy without sufficient information on the power. Even if the information on the powers is obtained enough, it is very hard to estimate the strategy, since the way to choose actions is very variable because of its dependency on time, the past states, the past rewards and so on. Therefore we treat only the information on the powers, which we call simply the information from now on. If a player obtains the information enough and knows the game structure, he can take an optimal strategy by means of the general game theory. Therefore, if the structure is not known and the game is played periodically, every player should treat it as the main subject to get the information as much as possible. We call such games the information games and study the amounts of information that players can obtain from sequential observations of actions, states and payoffs throughout all time.

We consider the information games as follows (see Fig. 1). In $n$-person games each player is called player $i$ and is denoted $P-i$. The power of each player is determined by some random system and is classified among finite grades $G=\left\{g_{1}, \cdots, g_{m}\right\}$ according to its quality and quantity. Every player does not know any other player's grade. A feasible action space of a player is determined according to his grade and is also not known to the others, so observed actions contain some information on the grades. Every player determine his strategy before the game begins. In the present paper we treat only simple strategies which are sequences of actions and are independent of history. At each time players' actions bring them some results such as rewards and states, called briefly states from now on. The states do not depend only on the actions but on the grades of powers. Thus the players observe the actions and the states, and obtain information on the grades. The main subject in this paper is to study how much 
amounts of information players can get from sequential observation of the actions and states through all time. We discuss the subject, applying the results of Rényi [11], [12], which treat the testing hypothesis theory and give some conditions under which the full information on a parameter can be obtained from sequential observations. Kai and Kanō [4] studied the subject in special games such that the power grades strictly determine the strategies, that is, the grades are essentially equivalent to the strategies. In this paper we treat generalized games and study relation between the information and the strategies.

In section 2 we discuss non-cooperative 2-person information games, where we give some sufficient conditions under which the full information on the grades can be obtained. At 2.2 we treat the cases that the states' sequences are Markov processes, at 2.3 and 2.4 study the cases that states' sequences are independent. At 2.4 we also show optimal strategies when the states are normally distributed. In section 3 we study n-person games, where we define the cooperation with respect to the information and show sufficient conditions under which the full information can be obtained by each of a cooperative group.

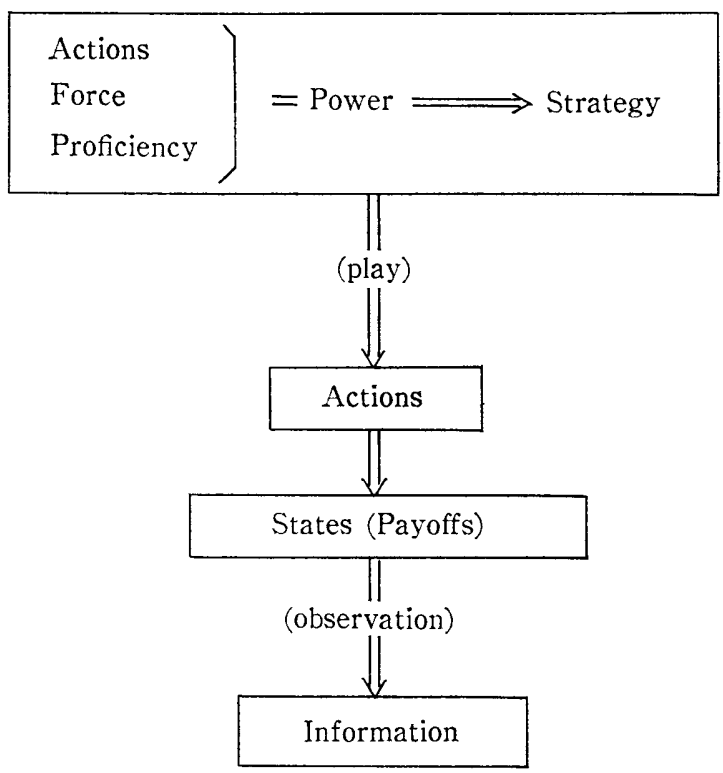

Fig. 1. Game system.

\section{2-person information games.}

\subsection{Introduction and essential theorems.}

2 -person information games are defined as follows. Let $G=\left\{g_{1}, \cdots, g_{m}\right\},(1 \leqq m<\infty\}$ be the set of all grades of powers. We suppose that the power of each player is determined by some random system, and let $P=\left\{p_{1}, \cdots, p_{m}\right\}$ be a probability on $G$ such that $P\left(\left\{g_{i}\right\}\right)=p_{i},(\geqq 0)$ and $\sum_{i=1}^{m} p_{i}=1$. Let $\theta_{1}$ and $\theta_{2}$ be parameters on $G$ which show 
the grades of $P-1$ 's power and $P$-2's power respectively, that is, $P\left(\theta_{1}=g_{i}\right)=P\left(\theta_{2}=g_{i}\right)$ $=p_{i}$. Let $A_{i}$ be the set of all feasible actions of a player whose grade is $g_{i}$. A player being in grade $g_{i}$ employs a strategy which is a sequence of actions $\delta_{i}=\left(\delta_{i 1}, \cdots, \delta_{i t}, \cdots\right)$ such that $\delta_{i t} \in A_{i}$ for all $t=1,2, \cdots$. We denote his grand strategy by the $m$-tuple of strategies $\delta=\left(\delta_{1}, \cdots, \delta_{m}\right)$, by which he takes $\delta_{i}$ if his grade is $g_{i}$. Let $S$ be the state space, assumed to be a Borel subset of a finite dimensional Euclidean space. Let $\xi_{t}=$ $\left(s_{0}, \cdots, s_{t}\right) \in S^{t+1}$ be the sequence of states up to time $t$, and let $\delta_{i}^{(t)}=\left(\delta_{i 1}, \cdots, \delta_{i t}\right)$ be the first $t$ actions of $\delta_{i}$. For a Borel subset $\Omega$, let $\mathfrak{B}(\Omega)$ be a $\sigma$-field of Borel subsets of $\Omega$.

Throughout this section we assume that for every grade $g_{i}$ of $P-1$ and $g_{j}$ of $P-2$ :

Al. The initial state $s_{0}$ is given by a probability measure $\Phi_{i j}^{(0)}(\cdot)$ on $\mathfrak{B}(S)$, assumed absolutely continuous, whose density is denoted by $\phi_{i j}^{(0)}\left(s_{0}\right)$;

A2. When $\delta_{i}$ and $\sigma_{j}$ were employed, $\xi_{t-1}$ occured, $\delta_{i t}$ and $\sigma_{j t}$ are taken, there exists a conditional probability $Q_{i j}^{(t)}\left(\cdot ; \xi_{t-1}, \delta_{i}^{(t)}, \sigma_{j}^{(t)}\right)$ on $\mathfrak{B}(S)$, i. e. for every $B \in \mathfrak{B}(S)$

$$
\begin{aligned}
\operatorname{Pr}\left\{s_{t}\right. & \left.\in B \mid \xi_{t-1}, \delta_{i}^{(t-1)}, \delta_{i l}, \sigma_{j}^{(t-1)}, \sigma_{j t}, \theta_{1}=g_{i}, \theta_{2}=g_{j}\right\} \\
& =Q_{i j}^{(t)}\left(B ; \xi_{t-1}, \delta_{i}^{(t)}, \sigma_{j}^{(t)}\right) ;
\end{aligned}
$$

A3. For every $t \geqq 1, i, j, \delta_{i}^{(t)}, \sigma_{j}^{(t)}$ and $\xi_{t-1}, Q_{i j}^{(t)}\left(\cdot ; \xi_{t-1}, \delta_{i}^{(t)}, \sigma_{j}^{(t)}\right)$ is absolutely continuous and let $q_{i j}^{(t)}\left(s_{t} \mid \xi_{t-1}, \delta_{i}^{(t)}, \sigma_{j}^{(t)}\right)$ be its density.

Let $\Phi_{i j}^{(t)}\left(\cdot ; \delta_{i}^{(t)}, \sigma_{j}^{(t)}\right)$ be a probability on $\mathfrak{B}\left(S^{t+1}\right)$ derived from $\Phi_{i j}^{(0)}(\cdot)$ and $\left\{Q_{i j}^{(L)}(\cdot\right.$; $\left.\left.\xi_{\nu-1}, \delta_{i}^{(\nu)}, \sigma_{j}^{(\nu)}\right) ;=1, \cdots, t\right\}$, i. e. for every $\prod_{\nu=0}^{t} B_{\nu} \in \mathfrak{B}\left(S^{t+1}\right)$

$$
\begin{aligned}
\Phi_{i j}^{(t)}\left(\prod_{\nu=0}^{t} B_{\nu} ; \delta_{i}^{(t)}, \sigma_{j}^{(t)}\right)= & \int_{B_{0}} \phi_{i j}^{(0)}\left(s_{0}\right) d s_{0} \int_{B_{1}} q_{i j}^{(1)}\left(s_{1} \mid \hat{\xi}_{c}, \delta_{i}^{(1)}, \sigma_{j}^{(1)}\right) d s_{1} \\
& \cdots \int_{B_{t}} q_{i j}^{(t)}\left(s_{t} \mid \hat{\xi}_{t-1}, \delta_{i}^{(t)}, \sigma_{j}^{(t)}\right) d s_{t} .
\end{aligned}
$$

Thus $\Phi_{i j}^{(t)}\left(\cdot ; \delta_{i}^{(t)}, \sigma_{j}^{(t)}\right)$ is absolutely continuous and has a density $\phi_{i j}^{(t)}\left(\xi_{t} ; \delta_{t}^{(t)}, \sigma_{j}^{(t)}\right)$ such that

$$
\phi_{i j}^{(t)}\left(\xi_{t} ; \delta_{i}^{(t)}, \sigma_{j}^{(t)}\right)=\phi_{i j}^{(0)}\left(s_{0}\right) \prod_{\nu=1}^{t} q_{i j}^{(\nu)}\left(s_{\nu} \mid \xi_{\nu-1}, \delta_{i}^{(\nu)}, \sigma_{j}^{(\nu)}\right) \text { a.s. }
$$

We suppose that both the players know all these systems.

Now we discuss the information about the grades of the players powers obtained from $\xi_{t}$. When $\theta_{1}=g_{i}$ and $\theta_{2}=g_{j}$, every player knows his own grade and up to time $t$ observes $\delta_{i}^{(t)}, \sigma_{j}^{(t)}$ and $\xi_{t}$, but he does not know the opponent's grade. To avoid complexity, we discuss the information from the side of $P-1$. Under each condition that $\theta_{1}=g_{i}, \theta_{2}=g_{j}, \delta_{i}$ and $\sigma_{j}$ are employed, the expected information on $\theta_{2}$ that $P-1$ can get from $\xi_{t}$ is defined by

$$
I_{1}^{(t)}\left(\delta_{i}, \sigma_{j}\right)=H\left(\theta_{2}\right)-E_{\tilde{o}_{i} \sigma_{j}}\left[H\left(\theta_{2} \mid \theta_{1}, \xi_{t}\right) \mid \theta_{1}=g_{i}, \theta_{2}=g_{j}\right]
$$

where $H\left(\theta_{2}\right)$ is the entropy of $\theta_{2}$ defined by Shannon's formula

$$
H\left(\theta_{2}\right)=\sum_{i=1}^{m}-p_{i} \log p_{i}
$$


$H\left(\theta_{2} \mid \theta_{1}, \xi_{t}\right)$ is the conditional entropy of $\theta_{2}$ when $\theta_{1}$ is known and $\xi_{t}$ is observed, i.e.

$$
H\left(\theta_{2} \mid \theta_{1}, \xi_{t}\right)=\sum_{k=1}^{m}-\operatorname{Pr}\left(\theta_{2}=g_{k} \mid \theta_{1}, \xi_{t}\right) \cdot \log \operatorname{Pr}\left(\theta_{2}=g_{k} \mid \theta_{1}, \xi_{t}\right),
$$

and $E_{\dot{o}_{i} \sigma_{j}}\left[H\left(\theta_{2} \mid \theta_{1}, \hat{\zeta}_{t}\right) \mid \theta_{1}=g_{i}, \theta_{2}=g_{j}\right]$ means the conditional expectation on the probability space $\left(S^{t+1}, \mathfrak{B}\left(S^{t+1}\right), \Phi_{i j}^{(t)}\left(\cdot ; \delta_{i}^{(t)}, \sigma_{j}^{(t)}\right)\right)$ with respect to $\theta_{1}=g_{i}$ and $\theta_{2}=g_{j}$, i.e.

$$
\begin{aligned}
& E_{\tilde{\delta}_{i} \sigma_{j}}\left[H\left(\theta_{2} \mid \theta_{1}, \xi_{t}\right) \mid \theta_{1}=g_{i}, \theta_{2}=g_{j}\right] \\
= & \int_{S^{t+1}}\left\{\sum_{k=1}^{m}-\operatorname{Pr}\left(\theta_{2}=g_{k} \mid \theta_{1}, \xi_{t}\right) \log \operatorname{Pr}\left(\theta_{2}=g_{k} \mid \theta_{1}, \xi_{t}\right)\right\} \cdot \dot{\phi}_{i j}^{(t)}\left(\xi_{t} ; \delta_{i}^{(t)}, \sigma_{j}^{(t)}\right) d \xi_{t} .
\end{aligned}
$$

Since a player in a grade $g_{i}$ can not take any action except the elements of $A_{i}$, one can define with no contradiction that

$$
\dot{\phi}_{k l}^{(t)}\left(\xi_{t} ; \delta_{i}^{(t)}, \sigma_{j}^{(t)}\right)=0 \quad \text { for all } \xi_{t} \in S^{t+1},
$$

if $\delta_{i}^{(t)} \notin A_{k}^{t}$ or $\sigma_{j}^{(t)} \notin A_{l}^{t}$. Therefore $\sigma_{j}^{(t)}$ contains some information on $\theta_{2}$. When $\theta_{1}=g_{i}$, $\delta_{i}^{(t)}$ and $\sigma_{j}^{(t)}$ are given, according to the Bayes' theorem and the theorems on conditional probability, we have from our assumptions that

$$
\begin{aligned}
& \operatorname{Pr}\left(\theta_{2}=g_{k} \mid \theta_{1}, \xi_{t}\right)=\operatorname{Pr}\left(\theta_{2}=g_{k} \mid \theta_{1}=g_{i}, \hat{\xi}_{t}\right) \\
= & \frac{p_{k} \phi_{i k}^{(t)}\left(\xi_{t} ; \delta_{l}^{(t)}, \sigma_{j}^{(t)}\right)}{\sum_{l} p_{l} \phi_{i l}^{(t)} \cdot\left(\xi_{t} ; \delta_{i}^{(t)}, \sigma_{j}^{(t)}\right)}
\end{aligned}
$$

Therefore, when the grand strategies $\delta=\left(\delta_{1}, \cdots, \delta_{m}\right)$ and $\sigma=\left(\sigma_{1}, \cdots, \sigma_{m}\right)$ are employed, $P-1$ can expect the following amount of information on $\theta_{2}$;

$$
\begin{aligned}
I_{1}^{(t)}(\tilde{\delta}, \sigma) & =E_{P}\left[I_{1}^{(t)}\left(\delta_{i}, \sigma_{j}\right)\right] \\
& =H\left(\theta_{2}\right)-\sum_{i} \sum_{j} p_{i} p_{j} E_{\delta_{i} \sigma_{j}}\left[H\left(\theta_{2} \mid \theta_{1}=\xi_{t}\right) \mid \theta_{1}=g_{i}, \theta_{2}=g_{j}\right] .
\end{aligned}
$$

It is easy to know that $I_{1}^{(t)}(\delta, \sigma)$ is nondecreasing for $t=1,2, \cdots$ and $I_{1}^{(t)}(\delta, \sigma) \leqq H\left(\theta_{2}\right)$. Thus $\lim _{t \rightarrow \infty} I_{1}^{(t)}(\delta, \sigma)=I_{1}^{(\infty)}(\delta, \sigma)$ alwayes exists. If $I_{1}^{(\infty)}(\delta, \sigma)=H\left(\theta_{2}\right)$, we say that $P-1$ obtains the full information on $\theta_{2}$ from the sequential observations $\left\{\xi_{t}\right\}, t=1,2, \cdots$.

We note a lemma of Rényi [11].

LEMMA 1. There exists a universal constant $C>0$ such that for any sequence $a_{1}, a_{2}, \cdots$, $a_{m}$ of positive numbers forming a probability distribution (i.e. $a_{1}+\cdots+a_{m}=1$ ) we have

$$
\sum_{i=1}^{m}-a_{i} \log a_{i} \leqq C \sum_{i=2}^{m} \sqrt{a_{i}} .
$$

Applying this lemma, we prove the following result.

LEMMA 2. There exists a constant $C$ such that for every $\delta, \sigma, i$ and $j$

$$
\begin{aligned}
& E_{\hat{o}_{i} \sigma_{j}}\left[H\left(\theta_{2} \mid \theta_{1}, \xi_{t}\right) \mid \theta_{1}=g_{i}, \theta_{2}=g_{j}\right] \\
\leqq & C \sum_{k \neq j} \int_{S^{t+1}}\left\{\frac{p_{k}}{p_{j}} \phi_{i j}^{(t)}\left(\xi_{t} ; \delta_{i}^{(t)}, \sigma_{j}^{(t)}\right) \phi_{i k}^{(t)}\left(\xi_{t} ; \delta_{i}^{(t)}, \sigma_{j}^{(t)}\right)\right\}^{112} d \xi_{t} .
\end{aligned}
$$


Proof. By Lemma 1 and (3), there exists a constant $C>0$ such that for any $1 \leqq$ $j \leqq m$

$$
H\left(\theta_{2} \mid \theta_{1}, \xi_{t}\right) \leqq C \sum_{k \neq j} \sqrt{P\left(\theta_{2}=g_{k} \mid \theta_{1}, \xi_{t}\right)} .
$$

Then from (4) and (5) it follows that

$$
\begin{aligned}
& E_{\hat{o}_{i} \sigma_{j}}\left[H\left(\theta_{2} \mid \theta_{1}, \xi_{t}\right) \mid \theta_{1}=g_{i}, \theta_{2}=g_{j}\right] \\
\leqq & C \sum_{k \neq j} \int_{S^{t+1}}\left\{\frac{p_{k} \phi_{i k}^{(t)}\left(\xi_{t} ; \delta_{i}^{(t)}, \sigma_{j}^{(t)}\right)}{\sum_{l} p_{l} \phi_{i l}^{(t)}\left(\xi_{t} ; \delta_{i}^{(t)}, \sigma_{j}^{(t)}\right)}\right\}^{1 / 2} \phi_{i j}^{(t)}\left(\xi_{t} ; \delta_{i}^{(t)}, \sigma_{j}^{(t)}\right) d \xi_{t} \\
\leqq & C \sum_{k \neq j} \int_{S^{t+1}}\left\{\frac{p_{k} \phi_{i k}^{(t)}\left(\xi_{t} ; \delta_{i}^{(t)}, \sigma_{j}^{(t)}\right)}{p_{j} \phi_{i j}^{(t)}\left(\xi_{t} ; \delta_{i}^{(t)}, \sigma_{j}^{(t)}\right.}\right\}^{1 / 2} \phi_{i j}^{(t)}\left(\xi_{t} ; \delta_{i}^{(t)}, \sigma_{j}^{(t)}\right) d \xi_{t} \\
= & C \sum_{k \neq j} \int_{S^{t+1}}\left\{\frac{p_{k}}{p_{j}} \phi_{i j}^{(t)}\left(\xi_{t} ; \delta_{i}^{(t)}, \sigma_{j}^{(t)}\right) \phi_{i k}^{(t)}\left(\xi_{t} ; \delta_{i}^{(t)}, \sigma_{j}^{(t)}\right)\right\}^{1 / 2} d \xi_{t} .
\end{aligned}
$$

THEOREM 1. If $\delta$ and $\sigma$ are employed and for every $i, j$, and $k \neq j$

$$
\lim _{t \rightarrow \infty} \int_{S^{t+1}}\left\{\phi_{i j}^{(t)}\left(\xi_{t} ; \delta_{i}^{(t)}, \sigma_{j}^{(t)}\right) \phi_{i k}^{(t)}\left(\xi_{t} ; \delta_{j}^{(t)}, \sigma_{j}^{(t)}\right)\right\}^{1 / 2} d \xi_{t}=0
$$

then $P-1$ obtains the full information on $\theta_{2}$, i.e. $I_{1}^{(\infty)}(\delta, \sigma)=H\left(\theta_{2}\right)$.

PROOF. By Lemma 2 it follows that for every $t \geqq 1$

$$
\begin{aligned}
& \sum_{i} \sum_{j} p_{i} p_{j} E_{\tilde{\sigma}_{i} \sigma_{j}}\left[H\left(\theta_{2} \mid \theta_{1}, \xi_{t}\right) \mid \theta_{1}=g_{i}, \theta_{2}=g_{j}\right] \\
& \quad \leqq C \sum_{i} \sum_{j} \sum_{k \neq j} p_{i} \sqrt{ } p_{j} p_{k}-\int_{S^{t+1}}\left\{\phi_{i j}^{(t)}\left(\xi_{t} ; \delta_{i}^{(t)}, \sigma_{j}^{(t)}\right) \phi_{i k}^{(t)}\left(\xi_{t} ; \delta_{i}^{(t)}, \sigma_{j}^{(t)}\right)\right\}^{1 / 2} d \xi_{t} .
\end{aligned}
$$

Then from (6) it implies the theorem.

\subsection{Sufficient conditions under which the full information is obtained in the case of Markov state-variables.}

We suppose that the states' sequence $\left\{s_{t} ; t=1,2, \cdots\right\}$ is a Markov process, that is, for every $i, j, t, a_{t} \in A, b_{t} \in A$ and $s_{t-1} \in S$ there exists a conditional probability $U_{i j}^{(t)}$ $\left(\cdot ; s_{t}, a_{t}, b_{t}\right)$ on $\mathfrak{B}(S)$ such that for every $B \in \mathfrak{B}(S), \delta_{i}^{(t-1)} \in A_{i}^{t-1}, \sigma_{j}^{(t-1)} \in A_{j}^{t-1}$ and $\xi_{t-2}$

$$
Q_{i j}^{(t)}\left(B \mid \xi_{t-2}, s_{t-1}, \delta_{i}^{(t-1)}, a_{t}, \sigma_{j}^{(t-1)}, b_{t}\right)=U_{i j}^{(t)}\left(s_{t} \in B ; s_{t-1}, a_{t}, b_{t}\right) .
$$

We denote the density of $U_{i j}^{(t)}\left(\cdot ; s_{t-1}, \delta_{i t}, \sigma_{j t}\right)$ by $u_{i j}^{(t)}\left(s_{t} ; s_{t-1}, \delta_{i t}, \sigma_{j t}\right)$, then the density $\phi_{i j}^{(t)}\left(\xi_{t} ; \delta_{i}^{(t)}, \sigma_{j}^{(t)}\right)$ of $\Phi_{i j}^{(t)}\left(\cdot ; \delta_{i}^{(t)}, \sigma_{j}^{(t)}\right)$ is a.s. equal to $\phi_{i j}^{(0)}\left(s_{0}\right) \prod_{\nu=1}^{t} u_{i j}^{(\nu)}\left(s_{\nu} ; s_{\nu-1}, \delta_{i \nu}, \sigma_{j \nu}\right)$. Denoting for each $t \geqq 1$

$$
\lambda_{i j k}^{(t)}\left(\delta_{i t}, \sigma_{j t}\right)=\sup _{s_{t-1}} \int_{S}\left\{u_{i j}^{(t)}\left(s_{t} ; s_{t-1}, \delta_{i t}, \sigma_{j t}\right) u_{i k}^{(t)}\left(s_{t} ; s_{t-1}, \delta_{i t}, \sigma_{j t}\right)\right\}^{1 / 2} d s_{t}
$$

we have the next result from Theorem 1 . 
THOREM 2. If $\delta$ and $\sigma$ are employed and for every $i, j$ and $k \neq j$

$$
\lim _{t \rightarrow \infty} \prod_{\nu=1}^{t} \lambda_{i j k}^{(\nu)}\left(\delta_{i \nu}, \sigma_{j \nu}\right)=0
$$

then $P-1$ obtains the full information on $\theta_{2}$.

PROOF. Since $\phi_{i j}^{(t)}\left(\xi_{t} ; \delta_{j}^{(t)}, \sigma_{j}^{(t)}\right)=\phi_{i j}^{(0)}\left(s_{0}\right) \prod_{\nu=1}^{\ell} u_{i j}^{(\nu)}\left(s_{\nu} ; s_{\nu-1}, \delta_{i \nu}, \sigma_{j \nu}\right)$ a. s., it follows that

$$
\begin{aligned}
& \int_{S^{t+1}}\left\{\phi_{i j}^{(t)}\left(\xi_{t} ; \delta_{i}^{(t)}, \sigma_{j}^{(t)}\right) \phi_{i k}^{(t)}\left(\xi_{t} ; \delta_{i}^{(t)}, \sigma_{j}^{(t)}\right)\right\}^{1 / 2} d \xi_{t} \\
& \quad=\int_{S^{t+1}} \phi_{i j}^{(0)}\left(s_{0}\right) \prod_{\nu=1}^{t}\left\{u_{i j}^{(\nu)}\left(s_{\nu} ; s_{\nu-1}, \delta_{i \nu}, \sigma_{j \nu}\right) u_{i k}\left(s_{\nu} ; s_{\nu-1}, \delta_{i \nu}, \sigma_{j \nu}\right)\right\}^{1 / 2} d \xi_{t} \\
& \quad \leqq \prod_{\nu=1}^{t} \lambda_{i j k}^{(\nu)}\left(\delta_{i \nu}, \sigma_{j \nu}\right)
\end{aligned}
$$

Thus by Theorem $1 P-1$ gets the full information on $\theta_{2}$.

We say that the Markov process is stationary if for every $a, b, s, s^{\prime}$ and $t, u_{i j}^{(t)}\left(s^{\prime}\right.$; $s, a, b)=u_{i j}^{(1)}\left(s^{\prime} ; s, a, b\right)$ a.s.

COROLlARY. If the states' sequence is a stationary Markov process and for every $i, j, k \neq j$

$$
\sup _{a \in A_{i} b \sqsubseteq A_{j}} \lambda_{i j k}^{(1)}(a, b)<1,
$$

then, employing any strategy, $P-1$ can get the full information on $\theta_{2}$.

Proof. By the assumption, for every $\delta, \sigma$ and $t$

$$
\begin{aligned}
\lambda_{i j k}^{(t)}\left(\delta_{i t}, \sigma_{j t}\right) & \leqq \sup _{a \in A_{i} b \in B_{j}} \lambda_{i j k}^{(1)}(a, b) \\
& <1
\end{aligned}
$$

then the result is derived by the theorem.

\subsection{Sufficient conditions under which the full information is obtained in the case of independent state-variables.}

We suppose that the states' sequence is independent, that is, for every $i, j, t, a \in A_{i}$ and $b \in A_{j}$ there exists a probability $V_{i j}^{(t)}(\cdot ; a, b)$ on $\mathfrak{B}(S)$ such that for every $B \in \mathfrak{B}(S)$, $\delta_{i}^{(t-1)}, \sigma_{j}^{(t-1)}$ and $\xi_{t-1}$

$$
Q_{i j}^{(t)}\left(B ; \xi_{t-1}, \delta_{i}^{(t-1)}, a, \sigma_{j}^{(t-1)}, b\right)=V_{i j}^{(t)}(B: a, b) .
$$

Denoting the density of $V_{i j}^{(t)}(\cdot ; a, b)$ by $f_{i j}^{(t)}\left(s_{t} ; a, b\right)$, one has

$$
\phi_{i j}^{(t)}\left(\xi_{t} ; \delta_{i}^{(t)}, \sigma_{j}^{(t)}\right)=\phi_{i j}^{(0)}\left(s_{0}\right) \prod_{\nu=1}^{t} f_{i j}^{(\nu)}\left(s_{\nu} ; \delta_{i \nu}, \sigma_{j \nu}\right) \quad \text { a.s. }
$$

Then we have the following result immediately from Theorem 1 . 
THEOREM 3. If $\delta$ and $\sigma$ are employed and for every $i, j$ and $k \neq j$

$$
\lim _{t \rightarrow \infty} \prod_{\nu=1}^{t} \int_{S}\left\{f_{i j}^{(\nu)}\left(s_{\nu} ; \delta_{i \nu}, \sigma_{j_{\nu}}\right) f_{i k}\left(s_{\nu} ; \delta_{i \nu}, \sigma_{j \nu}\right)\right\}^{1 / 2} d s_{\nu}=0
$$

then $P-1$ obtains the full information on $\theta_{2}$.

We show an application of this theorem.

ExPMPLE 1. Let $S=R^{2}, A_{i} \subset[0, \infty)$ for every $i$, and $f_{i j}^{(t)}\left(s_{t} ; \delta_{i t}, \sigma_{j t}\right)$ be a density of a 2-dimensional normal distribution, defined as follows;

$$
\begin{aligned}
& f_{i j}^{(t)}\left(x_{t}, y_{t} ; \delta_{i t}, \sigma_{j t}\right) \\
= & \frac{1}{2 \pi \delta_{i t} \sigma_{j t} \sqrt{ } 1-\rho_{t}^{2}} \exp \left[-\frac{1}{2\left(1-\rho_{t}^{2}\right)}\left\{\frac{\left(x_{t}-\alpha_{i j}\right)^{2}}{\delta_{i t}^{2}}\right.\right. \\
& \left.\left.-2 \rho_{t} \frac{\left(x_{t}-\alpha_{i j}\right)\left(y_{t}-\beta_{i j}\right)}{\delta_{i t} \sigma_{j t}}+\frac{\left(y_{t}-\beta_{i j}\right)^{2}}{\sigma_{j t}^{2}}\right\}\right] .
\end{aligned}
$$

Let $\alpha(t)_{i j k}=\frac{\alpha_{i j}-\alpha_{i k}}{\delta_{i t}}$ and $\beta(t)_{i j k}=\frac{\beta_{i j}-\beta_{i k}}{\sigma_{j t}}$. We suppose that $\rho_{t}$ is independent of $\delta_{i t}$ and $\sigma_{j t}$.

Proposition 1. Let $\delta$ and $\sigma$ be strategies of $P-1$ and $P-2$ respectively. If for every $i, j$ and $k \neq j$

$$
\sum_{t=1}^{\infty} \frac{1}{1-\rho_{t}^{2}}\left\{\alpha(t)_{i j k}^{2}-2 \rho_{t} \alpha(t)_{i j k} \beta(t)_{i j k}+\beta(t)_{i j k}^{2}\right\}=\infty
$$

then $P-1$ obtain the full information on $\theta_{2}$.

PROOF. Let $a_{i j k}=\frac{\alpha_{i j}+\alpha_{i k}}{2}$ and $b_{i j k}=\frac{\beta_{i j}+\beta_{i k}}{2}$. It follows

$$
\begin{aligned}
& \int_{R^{2}}\left\{f_{i j}^{(t)}\left(x_{t}, y_{t} ; \delta_{i t}, \sigma_{j t}\right) f_{i k}\left(x_{t}, y_{t} ; \delta_{i t}, \sigma_{j t}\right)\right\}^{1 / 2} d x_{t} d y_{t} \\
= & \frac{1}{2 \pi \delta_{i t} \sigma_{j t} \sqrt{1-\rho_{t}^{2}}} \exp \left[-\frac{1}{2\left(1-\rho_{t}^{2}\right)}\left\{\frac{\left(x_{t}-a_{i j k}\right)^{2}}{\delta_{i t}^{2}}\right.\right. \\
& -2 \rho_{t}-\frac{\left(x_{t}-a_{i j k}\right)\left(y_{t}-b_{i j k}\right)}{\delta_{i t} \sigma_{j t}}+\frac{\left(y_{t}-b_{i j k}\right)^{2}}{\sigma_{j t}^{2}} \\
& \left.\left.+\frac{1}{4} \alpha(t)_{i j k}^{2}-\frac{1}{2} \rho_{t} \alpha(t)_{i j k} \beta(t)_{i j k}+\frac{1}{4} \beta(t)_{i j k}^{2}\right\}\right] d x_{t} d y_{t} \\
= & \exp \left[-\frac{1}{8\left(1-\rho_{t}^{2}\right)}\left\{\alpha(t)_{i j k}^{2}-2 \rho_{t} \alpha(t)_{i j k} \beta(t)_{i j k}+\beta(t)_{i j k}^{2}\right\}\right] .
\end{aligned}
$$

Hence

$$
\begin{aligned}
& \prod_{\nu=1}^{t} \int_{R^{2}}\left\{f_{i j}^{(\nu)}\left(x_{\nu}, y_{\nu} ; \delta_{i \nu}, \sigma_{j \nu}\right) f_{i j}^{(\nu)}\left(x_{\nu}, y_{\nu} ; \delta_{i \nu}, \sigma_{j \nu}\right)\right\}^{1 / 2} d x_{\nu} d y_{\nu} \\
= & \exp \left\{-\frac{1}{8} \sum_{\nu=1}^{t} \begin{array}{c}
1-\rho_{\nu}^{2} \\
1
\end{array}\left(\alpha(\nu)_{i j k}^{2}-2 \rho_{\nu} \alpha(\nu)_{i j k} \beta(\nu)_{i j k}+\beta(\nu)_{i j k}^{2}\right)\right\}
\end{aligned}
$$


and we have the result by Theorem 2 .

REMARKS. Concerning the information on $\theta_{1}$ which may be obtained by $P-2$ we can prove the same results that we have shown with respect to the information on $\theta_{2}$ that $P-1$ can get. Defining the information that $P-2$ gets about $\theta_{1}$ by

$$
\begin{aligned}
& I_{2}^{(t)}(\delta, \sigma) \\
= & H\left(\theta_{1}\right)-\sum_{i} \sum_{j} p_{i} p_{j} E_{\hat{o}_{i} \sigma_{j}}\left[H\left(\theta_{1} \mid \theta_{2}, \xi_{t}\right) \mid \theta_{1}=g_{i}, \theta_{2}=g_{j}\right],
\end{aligned}
$$

one has:

THEOREM 1'. If $\delta$ and $\sigma$ are employed and for every $i, j$ and $k \neq i$

$$
\lim _{t \rightarrow \infty} \int_{S^{t+1}}\left\{\phi_{i j}^{(t)}\left(\xi_{t} ; \delta_{i}^{(t)}, \sigma_{j}^{(t)}\right) \phi_{k j}^{(t)}\left(\xi_{t} ; \delta_{i}^{(t)}, \sigma_{j}^{(t)}\right)\right\}^{1 / 2} d \xi_{t}=0
$$

then $P-2$ obtains the full information on $\theta_{1}$.

Both in independent case and in Markovian case we can show the same conditions under which $P-2$ obtains the full information on $\theta_{2}$.

\subsection{Optimal strategies when state-variables are independent and normally distributed.}

In the previous subsections we discussed the sufficient conditions under which a player can get the full information on the opponen's grade. However, every player should take account of both the information he gets and the information obtained by his opponent. Thus the object of $P-1$ in the game is to maximize the following quantity as soon as possible:

$$
I^{(t)}(\delta, \sigma)=I_{1}^{(t)}(\delta, \sigma)-I_{2}^{(t)}(\delta, \sigma) .
$$

Conversely $P-2$ wants to minimize the value of $I^{(t)}(\delta, \sigma)$ as soon as he can. For each time $t$ the system can be seen as a 2-person zero sum game, where the sets $\left\{\delta^{(t)} \equiv\left(\delta_{1}^{(t)}\right.\right.$, $\left.\left.\cdots, \delta_{m}^{(t)}\right)\right\}$ and $\left\{\sigma^{(t)} \equiv\left(\sigma_{1}^{(t)}, \cdots, \sigma_{m}^{(t)}\right)\right\}$ are action spaces of $P-1$ and $P-2$ respectively. Hence, for example, it every action space $A_{i}$ is finite, there exists the game value and exist optimal mixed strategies for both the players. Our problem in the present paper is to study optimal strategies through all time. We define $\delta^{*}$ and $\sigma^{*}$ to be optimal strategies for $P-1$ and $P-2$ respectively; if

$$
\begin{aligned}
\sup _{\tilde{\delta}} \inf _{\sigma} I^{(\infty)}(\delta, \sigma) & =\inf _{\sigma} \sup _{\tilde{\delta}} I^{(\infty)}(\delta, \sigma) \\
& =\inf _{\sigma} I^{(\infty)}\left(\delta^{*}, \sigma\right) \\
& =\sup _{\delta} I^{(\infty)}\left(\delta, \sigma^{*}\right),
\end{aligned}
$$

where $I^{(\infty)}(\delta, \sigma)=\lim _{t \rightarrow \infty} I^{(t)}(\delta, \sigma)$.

We suppose the states' sequence is independent, $S=R^{2}, A_{i}$ is a subset of $[0, \infty)$ for every $i$, and the probability density of $s_{t}=\left(x_{t}, y_{t}\right)$ is given by

$$
f_{i j}^{(t)}\left(x_{t}, y_{t} ; \delta_{i t}, \sigma_{j t}\right)
$$




$$
\begin{aligned}
= & -\frac{1}{2 \pi \delta_{i t} \sigma_{j t} \sqrt{1-\rho_{t}^{2}}} \exp \left[-\frac{1}{2\left(1-\rho_{t}^{2}\right)}\left\{\frac{\left(x_{t}-u_{i}\right)^{2}}{\delta_{i t}^{2}}\right.\right. \\
& \left.\left.-2 \rho_{t}-\frac{\left(x_{t}-u_{i}\right)\left(y_{t}-v_{j}\right)}{\delta_{i t} \sigma_{j t}}+\frac{\left(y_{t}-v_{j}\right)^{2}}{\sigma_{j t}^{2}}\right\}\right] .
\end{aligned}
$$

LEMMA 3. If $\rho_{t}=\frac{\operatorname{cov}\left(x_{t}, y_{t}\right)}{\delta_{i t} \sigma_{j t}}$ is independent of $\delta_{i t}$ and $\sigma_{j t}$, then $I_{1}^{(t)}(\delta, \sigma)$ is independent of $\delta$.

ProOF. For every $i$ anf $j$

$$
\begin{aligned}
& E_{\tilde{\delta}_{i} \sigma}\left[H\left(\theta_{2} \mid \theta_{1}, \xi_{t}\right) \mid \theta_{1}=g_{i}, \theta_{2}=g_{j}\right] \\
& \quad=\int_{R^{2 t}}\left\{\sum_{k} \frac{p_{k} \phi_{i k}^{(t)}\left(\xi_{t} ; \delta_{i}^{(t)}, \sigma_{j}^{(t)}\right)}{\sum_{l} p_{l} \phi_{i l}^{(t)}\left(\xi_{t} ; \delta_{i}^{(t)}, \sigma_{j}^{(t)}\right)} \log \frac{\sum_{l} p_{l} \phi_{i l}^{(t)}\left(\xi_{t} ; \delta_{i}^{(t)}, \sigma_{j}^{(t)}\right)}{p_{k} \phi_{i k}^{(t)}\left(\xi_{t} ; \delta_{i}^{(t)}, \sigma_{j}^{(t)}\right)}\right\} \cdot \phi_{i j}^{(t)}\left(\xi_{t} ; \delta_{i}^{(t)}, \sigma_{j}^{(t)}\right) d \xi_{t},
\end{aligned}
$$

where

$$
\phi_{i l}^{(t)}\left(\xi_{t}: \delta_{i}^{(t)}, \sigma_{j}^{(t)}\right)=\phi_{i l}^{(0)}\left(x_{0}, y_{0}\right) \prod_{\nu=1}^{t} f_{i l}^{(\nu)}\left(x_{\nu}, y_{\nu} ; \delta_{i \nu}, \sigma_{j_{\nu}}\right)
$$

From (11), letting $x_{\nu} \equiv \frac{x_{\nu}-u_{i}}{\delta_{i_{\nu}}}$ for every $\nu$, we see $E_{\tilde{o}_{i} \sigma_{j}}\left[H\left(\theta_{2} \mid \theta_{1}, \xi_{t}\right) \mid \theta_{1}=g_{i}, \theta_{2}=g_{j}\right]$ is independent of $\delta$.

If a grand strategy does not depend on powers' grades, i. e. $\delta_{1}=\cdots=\delta_{m}$, or $\sigma_{1}=\cdots$ $=\sigma_{m}$, we call it "an identical strategy".

THEOREM 4. Let us suppose that for every $t, \delta$ and $\sigma, \rho_{t}$ is independent of $\delta_{i t}$ and $\sigma_{j t}$. Then for identical strategies $\delta$ and $\delta^{\prime}$ such as $\delta_{1 t} \leqq \delta_{1 t}^{\prime}$, for all $t \geqq 1$, we have

$$
I^{(t)}(\delta, \sigma) \leqq I^{(t)}\left(\delta^{\prime}, \sigma\right) .
$$

Proof. From the assumption and Lemma $3, I_{1}^{(t)}(\delta, \sigma)$ is independent of $\delta$. Thus we treat only $I_{2}^{(t)}(\delta, \sigma)$. Denote $\delta_{0}=\delta_{1}$. Since $\delta_{1}=\cdots=\delta_{m}$, we have

$$
\begin{aligned}
& \sum_{i} p_{i} E_{\delta_{i} \sigma_{j}}\left[H\left(\theta_{1} \mid \theta_{2}, \xi_{t}\right) \mid \theta_{1}=g_{i}, \theta_{2}=g_{j}\right] \\
&=\sum_{i} p_{i} \int_{R^{2(t+1)}} \sum_{k}\left\{\frac{p_{k} \phi_{k j}^{(t)}\left(\xi_{t} ; \delta_{0}^{(t)}, \sigma_{j}^{(t)}\right)}{\sum_{l} p_{l} \phi_{l j}^{(t)}\left(\xi_{t} ; \delta_{0}^{(t)}, \sigma_{j}^{(t)}\right)} \log \frac{\sum_{l} p_{l} \phi_{l j}^{(t)}\left(\xi_{t} ; \delta_{0}^{(t)}, \sigma_{j}^{(t)}\right)}{p_{k} \phi_{k j}^{(t)}\left(\xi_{t} ; \delta_{0}^{(t)}, \sigma_{j}^{(t)}\right)}\right\} \\
& \cdot \phi_{i j}^{(t)}\left(\xi_{t} ; \delta_{0}^{(t)}, \sigma_{j}^{(t)}\right) d \xi_{t} \\
&= \sum_{k} \int_{R^{2}(t+1)}\left\{p_{k} \phi_{k j}^{(t)}\left(\xi_{t} ; \delta_{0}^{(t)}, \sigma_{j}^{(t)}\right) \log \frac{p_{k} \phi_{k j}^{(t)}\left(\xi_{t} ; \delta_{0}^{(t)}, \sigma_{j}^{(t)}\right)}{\sum_{l} p_{l} \phi_{l j}^{(t)}\left(\xi_{t} ; \delta_{0}^{(t)}, \sigma_{j}^{(t)}\right)}\right\} d \xi_{t} .
\end{aligned}
$$

Putting $x_{2} \equiv \frac{x_{2}-u_{i}}{\delta_{o \nu}}$ and $y_{2} \equiv \frac{y_{\nu}-\sigma_{j}}{\sigma_{j \nu}}$ for every $\nu, 1 \leqq \nu \leqq t$, we get

$$
\sum_{i} p_{i} E_{\tilde{o}_{i} \sigma_{j}}\left[H\left(\theta_{1} \mid \theta_{2}, \xi_{t}\right) \mid \theta_{1}=g_{i}, \theta_{2}=g_{j}\right]
$$




$$
\begin{aligned}
& =\sum_{k} \int_{R^{2}} \frac{p_{k} \phi_{k j}^{(0)}\left(x_{0}, y_{0}\right)}{2 \pi \sqrt{1-\rho_{0}^{2}}} d x_{0} d y_{0} \\
& \cdot \int_{R^{2 t}} \prod_{\nu=1}^{t} \exp \left\{\frac{-1}{2\left(1-\rho_{\nu}^{2}\right)}\left(x_{\nu}^{2}-2 \rho_{\nu} x_{\nu} y_{\nu}+y_{\nu}^{2}\right)\right\} \\
& \cdot \log \left\{\sum_{t}-\frac{p_{l}}{p_{k}} \prod_{\nu=1}^{t} \exp \frac{u_{l}-u_{k}}{\delta_{02}\left(1-\rho_{\nu}^{2}\right)}\left(x_{\nu}-\frac{u_{l}-u_{k}}{2 \delta_{0 \nu}}-\rho_{\nu} y_{\nu}\right)\right\} d \xi_{t} .
\end{aligned}
$$

Denoting the last integral on $R^{2 t}$ by $F_{k j}^{(t)}\left(\delta_{0}, \sigma_{j}\right)$, we get for every $n, 1 \leqq n \leqq t$,

$$
\begin{aligned}
& \frac{\partial F_{k j}^{(t)}\left(\delta_{0}, \sigma_{j}\right)}{\partial \delta_{0 n}} \\
&= \int_{R^{2}} \prod_{\nu=1}^{t} \exp \left\{\frac{-1}{2\left(1-\rho_{\nu}^{2}\right)}\left(x_{\nu}^{2}-2 \rho_{\nu} x_{\nu} y_{\nu}+y_{\nu}^{2}\right)\right\} \\
& \cdot\left\{\sum_{l} \frac{p_{l}\left(u_{l}-u_{k}\right)}{p_{k}\left(1-\rho_{\nu}^{2}\right) \delta_{0 n}^{2}}\left(\frac{u_{l}-u_{k}}{\delta_{0 n}}-x_{\nu}+\rho_{\nu} y_{\nu}\right)\right. \\
&\left.\cdot \prod_{\nu=1}^{t} \exp \frac{u_{l}-u_{k}}{\delta_{0 \nu}\left(1-\rho_{\nu}^{2}\right)}\left(x_{\nu}-\frac{u_{l}-u_{k}}{2 \delta_{0 \nu}}-\rho_{\nu} y_{\nu}\right)\right\} \\
& / \sum_{l} \frac{p_{l}}{p_{k}} \prod_{\nu=1}^{t} \exp \frac{u_{l}-u_{k}}{\delta_{0 \nu}\left(1-\rho_{\nu}^{2}\right)}\left(x_{\nu}-\frac{u_{l}-u_{k}}{2 \delta_{0 \nu}}-\rho_{\nu} y_{\nu}\right) d \xi_{t} \\
&= \frac{1}{\delta_{0 n}} \int_{R^{2 t}}\left\{\sum_{l} \frac{p_{l} c_{l k}^{(n)}}{1-\rho_{n}^{2}}\left(c_{l k}^{(n)}-x_{n}+\rho_{n} y_{n}\right)\right. \\
& \cdot\left.\prod_{\nu=1}^{t} \exp \frac{-1}{2\left(1-\rho_{\nu}^{2}\right)}\left(\left(x_{\nu}-c_{l k}^{(\nu)}\right)^{2}-2 \rho_{\nu} y_{\nu}\left(x_{\nu}-c_{l k}^{(\nu)}\right)+y_{\nu}\right)\right\} \\
& / \sum_{l} p_{l} \prod_{\nu=1}^{t} \exp \frac{c_{l k}^{(\nu)}}{1-\rho_{\nu}^{2}}\left(x_{\nu}-\frac{c_{l k}^{(\nu)}}{2}-\rho_{\nu} y_{\nu}\right) d \xi_{t},
\end{aligned}
$$

where $c_{l k}^{(L)}=\frac{u_{l}-u_{k}}{\delta_{0 \nu}}$. Then it follows

$$
\begin{aligned}
& \frac{\partial F_{i j}^{(t)}\left(\delta_{0}, \sigma_{j}\right)}{\partial \delta_{0 n}} \\
= & \frac{1}{\delta_{0 n}} \int_{R^{2 t}} \sum_{l} p_{l} c_{l k}^{(n)} \\
& \cdot \prod_{\nu=1}^{t} \exp \left\{\frac{-1}{2\left(1-\rho_{\nu}^{2}\right)}\left(\left(x_{\nu}-c_{l k}^{(\nu)}\right)^{2}-2 \rho_{\nu} y_{\nu}\left(x_{\nu}-c_{l k}^{(k)}\right)+y_{\nu}^{2}\right)\right\} \\
\cdot & \left\{\sum_{l} p_{l} c_{l k}^{(n)} \rho_{n}^{t} \prod_{\nu=1}^{t} \exp \frac{c_{l k}^{(\nu)}}{1-\rho_{\nu}^{2}}\left(x_{\nu}-\frac{c_{l k}^{(L)}}{2}-\rho_{\nu} y_{\nu}\right)\right\} \\
& /\left\{\sum_{l} p_{l} \prod_{\nu=1}^{t} \exp \frac{c_{l k}^{(\nu)}}{1-\rho_{\nu}^{2}}\left(x_{\nu}-\frac{c_{l k}^{(\nu)}}{2}-\rho_{\nu} y_{\nu}\right)\right\}^{2} d \hat{\zeta}_{t} \\
\geqq & 0 .
\end{aligned}
$$


Hence for every $j$ and $1 \leqq n \leqq t$

$$
-\frac{\partial}{\partial \delta_{0 n}}\left\{\sum_{i} E_{\tilde{o}_{0} \sigma_{j}}\left[H\left(\theta_{1} \mid \theta_{2}, \xi_{t}\right) \mid \theta_{1}=g_{i}, \theta_{2}=g_{j}\right]\right\} \geqq 0 .
$$

Thus from (6)

$$
\frac{\partial}{\partial \delta_{0 n}} I^{(t)}(\delta, \sigma) \geqq 0
$$

for all $1 \leqq n \leqq t$, which implies the theorem.

THEOREM 5. Let us suppose that $A_{1}=\cdots=A_{m}=[\alpha, \beta] \subset[0, \infty)$ and both the players take only identical strategies. If for every $t, \delta_{i}$ and $\sigma_{j}, \rho_{t}$ is independent of $\delta_{i t}$ and $\sigma_{j t}$, then a strategy $\delta_{0}^{*}=\left(\delta_{0}^{*}, \cdots, \delta_{0}^{*}\right), \delta_{0 t}^{*}=\beta$ for all $t \geqq 1$, is optimal for both the players.

Proof. By Lemma 3 and Theorem 4, for any identical strategies $\delta$ and $\sigma$ we have

$$
I^{(t)}\left(\delta, \hat{\delta}_{0}^{*}\right) \leqq I^{(t)}\left(\delta_{0}^{*}, \delta_{0}^{*}\right) \leqq I^{(t)}\left(\delta_{0}^{*}, \sigma\right) \quad \text { for all } t .
$$

Thus, since for every $\delta$ and $\sigma, I^{(t)}(\delta, \sigma)$ converges to $I^{(\infty)}(\delta, \sigma)$, we hold

$$
I^{(\infty)}\left(\delta, \delta_{0}^{*}\right) \leqq I^{(\infty)}\left(\delta_{0}^{*}, \delta_{0}^{*}\right) \leqq I^{(\infty)}\left(\delta_{0}^{*}, \sigma\right),
$$

which implies the theorem.

\section{3. n-person information games.}

\subsection{Definition.}

We define $n$-person information games which are extension of 2-person information games. Let $N=\{1, \cdots, n\}$ be the set of players, $G=\left\{g_{1}, \cdots, g_{m}\right\}$ be the set of grades with respect to the powers, $P=\left(p_{1}, \cdots, p_{m}\right)$ be a probability on $G$ such that $P\left(g_{i}\right)=p_{i}$ and $\sum_{i} p_{i}=1, S$ be the state space which is assumed to be Borel subset of a finite dimensional Euclidean space, and $A_{j},(i=1, \cdots, m)$ be the set of all feasible actions of a player being in grade $g_{i}$. Let $\theta_{l},(l=1, \cdots, n)$ be a parameter on $G$ which shows the grade of the $l$-th player $P-l$. A stretegy of $P-l$ being in grade $g_{i}$ is a sequence of actions $\delta_{i}(l)=\left(\delta_{i}(l)_{1}, \delta_{i}(l)_{2}, \cdots, \delta_{i}(l)_{t}, \cdots\right)$, where $\delta_{i}(l)_{t}$ is an element of $A_{i}$ for all $t$. Let $\delta_{i}^{(t)}(l)=\left(\delta_{i}(l)_{1}, \cdots, \delta_{i}(l)_{t}\right)$ be the first $t$ subsequence of $\delta_{i}(l)$ and let $\delta(l)=\left(\delta_{1}(l), \cdots\right.$, $\left.\delta_{m}(l)\right)$ be a grand strategy of $P-l$. Let $g_{i_{l}},(l=1, \cdots, n)$ be a grade of $P$-l's power and denote $m$-tuple of their strategies by $\delta\left(i_{1}, \cdots, i_{n}\right)=\left(\delta_{i_{1}}(1), \cdots, \delta_{i_{n}}(n)\right)$ and denote $\delta^{(t)}\left(i, \cdots, i_{n}\right)=\left(\delta_{i_{1}}^{(t)}(l), \cdots, \delta_{i_{n}}^{(t)}(n)\right)$. Let $\xi_{t}=\left(s_{0}, \cdots, s_{t}\right) \in S^{t+1}$ be states' sequence up to time $t$.

We suppose; when players' grade are $g_{i_{1}}, \cdots, g_{i_{n}}, \delta^{(t)}\left(i_{1}, \cdots i_{n}\right)$ are employed and $\xi_{t}$ is observed, a state $s_{t}$ at time $t$ is determined a conditional probability $Q_{i_{1} \ldots i_{n}}^{(t)}\left(\cdot ; \delta^{(t)}\right.$ $\left.\left(i_{1}, \cdots, i_{n}\right)\right)$ on $\mathfrak{B}(S)$, which is assumed to be absolutely continuous. Let $\Phi_{i_{1} \ldots i_{n}}^{(0)}(\cdot)$ be a probability on $\mathfrak{B}(S)$, which determines the initial state $s_{0}$ and is assumed to be absolutely continuous. Thus a probality $\Phi_{i_{1} \ldots i_{n}}^{(t)}\left(\cdot ; \delta^{(t)}\left(i_{1}, \cdots, i_{n}\right)\right)$ on $\mathfrak{B}\left(S^{t+1}\right)$ is derived from $\Phi_{i_{1} \ldots i_{n}}^{(0)}(\cdot)$ and $\left\{Q_{i_{1} \ldots i_{n}}^{(\nu)}\left(\cdot ; \delta^{(\nu)}\left(i_{1}, \cdots, i_{n}\right)\right) ; \nu=1, \cdots, t\right\}$. From the assumptions each $\Phi_{i_{1} \cdots i_{n}}^{(t)}$ $\left(\cdot ; \delta^{(t)}\left(i_{1}, \cdots, i_{n}\right)\right)$ has a density, then we denote it by $\phi_{i_{1} \cdots i_{n}}^{(t)}\left(\xi_{t} ; \delta^{(t)}\left(i_{1}, \cdots, i_{n}\right)\right)$. Since every player can not take any action except the elements of feasible actions' set restricted by his power, we define that 


$$
\dot{\phi}_{i_{1} \cdots i_{l-1}{ }^{j i} i_{l+1} \cdots i_{n}}^{(t)}\left(\xi_{t} ; \delta^{(t)}\left(i_{1}, \cdots, i_{n}\right)\right)=0
$$

for all $\xi_{t}$ if an observed sequence of actions $\delta_{i l}^{(t)}(l)$ of $P-l$ is not an element of $A_{j}^{t}$.

To avoid complexity, we treat only $P-1$ 's information about $P-n$ 's power. When players' grades are $g_{i_{1}}, \cdots, g_{i_{n}}$ and $\delta_{i_{1}}(1), \cdots, \delta_{i_{n}}(n)$ are employed, the expected information that $P-1$ obtains about $\theta_{n}$ is defined by

where

$$
\begin{aligned}
& I_{1}^{(t)}\left(\theta_{n}: \delta\left(i_{1}, \cdots, i_{n}\right)\right) \\
= & H\left(\theta_{n}\right)-E_{\partial^{\prime}\left(i_{1}, \cdots, i_{n}\right)}\left[H\left(\theta_{n} \mid \theta_{1}, \xi_{t}\right) \mid \theta_{1}=g_{i_{1}}, \cdots, \theta_{n}=g_{i_{n}}\right],
\end{aligned}
$$

$$
E_{\partial\left(i_{1}, \ldots, i_{n}\right.}:\left[H\left(\theta_{n} \mid \theta_{1}, \xi_{t}\right) \mid \theta_{1}=g_{i_{1}}, \cdots, \theta_{n}=g_{i_{n}}\right]
$$

is the conditional expectation of $H\left(\theta_{n} \mid \theta_{1}, \xi_{t}\right)$ given $g_{i_{1}}, \cdots, g_{i_{n}}$ with respect to the probability $\Phi_{i_{1}}^{(t)}, \ldots, i_{n}\left(\cdot ; \delta^{(t)}\left(i_{1}, \cdots, i_{n}\right)\right)$, and $H\left(\theta_{n} \mid \theta_{1}, \xi_{t}\right)$ is a conditional entropy of $\theta_{n}$ given $\theta_{1}$ and $\xi_{t}$, i. e.

$$
H\left(\theta_{n} \mid \theta_{1}, \hat{\xi}_{t}\right)=\sum_{\boldsymbol{j}}-\operatorname{Pr}\left(\theta_{n}=g_{j} \mid \theta_{1}, \hat{\xi}_{t}\right) \log \operatorname{Pr}\left(\theta_{n}=g_{j} \mid \theta_{1}, \xi_{t}\right) .
$$

According to the Bayes' theorem and the theorems on conditional probability, we have for $\theta_{1}=g_{i_{1}}$ and $\delta^{(t)}\left(i_{1}, \cdots, i_{n}\right)$

$$
\operatorname{Pr}\left(\theta_{n}=g_{j_{n}} \mid \theta_{1}=g_{i_{1}}, \xi_{t}\right)=\frac{p_{j_{n}} \psi_{i_{1} j(t)}^{(t)}\left(\xi_{t} ; \delta^{(t)}\left(i_{1}, \cdots, i_{n}\right)\right)}{\sum_{l_{n}} p_{l_{n}} \phi_{i_{1} l_{n}}^{(t)}\left(\xi_{t} ; \delta^{(t)}\left(i_{1}, \cdots, i_{n}\right)\right)},
$$

where

$$
\begin{aligned}
& \dot{\varphi}_{i_{1} l_{n}}^{(t)}\left(\xi_{t} ; \delta^{(t)}\left(i_{1}, \cdots, i_{n}\right)\right) \\
= & \sum_{j_{2}} \cdots \sum_{j_{n-1}} p_{j_{2}} \cdots p_{j_{n-1}} \phi_{i_{1} j_{2} \cdots j_{n-1} l_{n}}^{\left(\xi_{t}\right.}\left(\xi^{(t)}\left(i_{1}, \cdots, i_{n}\right)\right),
\end{aligned}
$$

which is a conditional probability density on $\mathfrak{B}\left(S^{t+1}\right)$ when $\theta_{1}=g_{i_{1}}$ and $\theta_{n}=g_{l_{n}}$ are given and $\delta^{(t)}\left(i_{1}, \cdots, i_{n}\right)$ is observed. Hence we get

$$
\begin{aligned}
& E_{\hat{\sigma}\left(i_{1}, \cdots, i_{n}\right.}:\left[H\left(\theta_{n} \mid \theta_{1}, \xi_{t}\right) \mid \theta_{1}=g_{i_{1}}, \cdots, \theta_{n}=g_{i_{n}}\right] \\
& =\int_{S^{t+1}} \sum_{j_{n}}\left\{\begin{array}{c}
p_{j_{n}} \dot{\psi}_{i_{1} j_{n}}^{(t)}\left(\xi_{t} ; \delta^{(t)}\left(i_{1}, \cdots, i_{n}\right)\right) \\
\sum_{l_{n}} p_{l_{n}} \dot{\psi}_{i_{1} l_{n}}^{(t)}\left(\xi_{t} ; \delta^{(t)}\left(i_{1}, \cdots, i_{n}\right)\right)
\end{array}\right. \\
& \left.\cdot \log \frac{\sum_{l_{n}} \frac{p_{l_{n}} \phi_{i_{1} l_{n}}^{(t)}\left(\xi_{t} ; \delta^{\langle t\rangle}\left(i_{1}, \cdots, i_{n}\right)\right)}{p_{j_{n}} \phi_{i_{1} j_{n}}^{(t)}\left(\xi_{t} ; \delta^{\langle t\rangle}\left(\overline{i_{1}}, \cdots, i_{n}\right)\right)}}{\}}\right\} \\
& \text { - } \left.\dot{\phi}_{\hat{\delta}\left(i_{1}, \cdots, i_{n}\right)}^{\left(\xi_{t}\right.} ; \delta^{(t)}\left(i_{1}, \cdots, i_{n}\right)\right) d \xi_{t} \text {. }
\end{aligned}
$$

Therefore the expected information on $\theta_{n}$ obtained by $P-1$ with respect to the grand strategies $\delta(1), \cdots, \delta(n)$ is defined by

$$
\begin{aligned}
& I_{1}^{(t)}\left(\theta_{n} ; \delta(1), \cdots, \delta(n)\right) \\
= & E_{p}\left[I_{1}^{(t)}\left(\theta_{n} ; \delta\left(i_{1}, \cdots, i_{n}\right)\right)\right] \\
= & H\left(\theta_{n}\right)-\sum_{i_{1}} \cdots \sum_{i_{n}} p_{i_{1}} \cdots p_{i_{n}} \\
& \quad E_{\delta\left(i_{1}, \cdots, i_{n}\right)}\left[H\left(\theta_{n} \mid \theta_{1}, \xi_{t}\right) \mid \theta_{1}=g_{i_{1}}, \cdots, \theta_{n}=g_{i_{n}}\right] .
\end{aligned}
$$


It is easy to show that $I_{1}^{(t)}\left(\theta_{n} ; \delta(1), \cdots, \delta(n)\right)$ is nondecreasing for $t=1,2, \cdots$ and $I_{1}^{(t)}\left(\theta_{n} ; \delta(1), \cdots, \delta(n)\right) \leqq H\left(\theta_{n}\right)$. Hence $\lim _{t \rightarrow \infty} I_{1}^{(t)}\left(\theta_{n} ; \delta(1), \cdots, \delta(n)\right)=I_{1}^{(\infty)}\left(\theta_{n} ; \delta(1), \cdots, \delta(n)\right)$ always exists. We say that $P-1$ gets the full information on $\theta_{n}$ if $I_{1}^{(\infty)}\left(\theta_{n} ; \delta(1), \cdots\right.$, $\delta(n))=H\left(\theta_{n}\right)$. We show a sufficient condition under which the full information is obtained.

THEOREM 6. If for every $i_{1}, \cdots, i_{n}$ and $j_{n} \neq i_{n}$

$$
\lim _{t \rightarrow \infty} \int_{S^{t+1}}\left\{\dot{\psi}_{1_{1} i_{n}}^{(t)}\left(\xi_{t} ; \delta^{(t)}\left(i_{1}, \cdots, i_{n}\right)\right) \psi_{i_{1} j_{n}}^{(t)}\left(\xi_{t} ; \delta^{(t)}\left(i_{1}, \cdots, i_{n}\right)\right)\right\}^{1 \backslash 2} d \xi_{t}=0
$$

then $P-1$ obtains the full information on $\theta_{n}$.

Proof. By Lemma 1 there exists a constant $C>0$ such that

$$
\begin{aligned}
& E_{\partial\left(i_{1} \cdots i_{n}\right)}\left[H\left(\theta_{n} \mid \theta_{1}, \xi_{t}\right) \mid \theta_{1}=g_{i_{1}}, \cdots, \theta_{n}=g_{i_{n}}\right] \\
\leqq & \int_{S^{t+1}} \sum_{j_{n} \neq i_{n}}\left\{\operatorname{Pr}\left(\theta_{n}=g_{j_{n}} \mid \theta_{1}=g_{i_{1}}, \xi_{t}\right)\right\}^{1 / 2} \cdot \phi_{i_{1} \cdots i_{n}}^{(t)}\left(\hat{\xi}_{t} ; \delta^{(t)}\left(i_{1}, \cdots, i_{n}\right)\right) d \hat{\xi}_{t} .
\end{aligned}
$$

Since

$$
p_{i_{2}} \cdots p_{i_{n-1}} \phi_{i_{1} \cdots i_{n}}^{(t)}\left(\xi_{t} ; \delta^{(t)}\left(i_{1}, \cdots, i_{n}\right)\right) \leqq \phi_{i_{1} i_{n}}^{(t)}\left(\xi_{t} ; \delta^{(t)}\left(i_{1}, \cdots, i_{n}\right)\right),
$$

from (12) and (14) we obtain the conclusion.

Applying this theorem we can hold sufficient conditions similar as Theorem 2 and Theorem 3 under which the full information is obtained when the states' sequence is Markovian or is independent.

\subsection{Cooperation with respect to information.}

Let $K=\{1, \cdots, k\} \subset N$ be a cooperative group. Considering the cooperation between the players in $K$, we must not treat only the strategies to be employed, but take account of the information they have. Even though they cooperate each other in some way, they can hardly map out effective strategies without enough information about every player in the group. Therefore we discuss the cooperation from the viewpoint of information and study conditions under which the full information on the opponents to the cooperative group is obtained. We define that the cooperation in a group is to offer each other all information they have about the others and themselves. In the present paper the cooperation is defined to offer the information on tneir own power, then every player in the group knows the powers of other members. We define the information each of $K$ gets about $\theta_{n}$ under the cooperation as follows: for $\delta(1), \cdots, \delta(n)$

$$
\begin{aligned}
& I_{K}^{(t)}\left(\theta_{n} ; \delta(1), \cdots, \delta(n)\right) \\
= & H\left(\theta_{n}\right)-E_{P}\left[E_{\tilde{o}\left(i_{1}, \cdots, i_{n}\right)}\left[H\left(\theta_{n} \mid \theta_{1}, \cdots, \theta_{k}, \xi_{t}\right) \mid \theta_{1}=g_{i_{1}}, \cdots, \theta_{n}=g_{i_{n}}\right]\right],
\end{aligned}
$$

where

$$
\begin{aligned}
& E_{\partial\left\langle i_{1} \cdots, i_{n}\right)}\left[H\left(\theta_{n} \mid \theta_{1}, \cdots, \theta_{k}, \xi_{t}\right) \mid \theta_{1}=g_{i_{1}}, \cdots, \theta_{n}=g_{i_{n}}\right] \\
= & \int_{S^{t}+1} \sum_{j_{n}}\left\{-\operatorname{Pr}\left(\theta_{n}=g_{i_{n}} \mid \theta_{1}=g_{i_{1}}, \cdots, \theta_{k}=g_{i_{k}}, \hat{\xi}_{t}\right)\right. \\
& \left.\cdot \log \operatorname{Pr}\left(\theta_{n}=g_{i_{n}} \mid \theta_{1}=g_{i}, \cdots, \theta_{k}=g_{i_{k}}, \xi_{t}\right)\right\} \dot{\phi}_{i_{1} \cdots i_{n}}^{(t)}\left(\xi_{t} ; \delta^{(t)}\left(i_{1}, \cdots, i_{n}\right)\right) d \hat{\xi}_{t}
\end{aligned}
$$


and

$$
\operatorname{Pr}\left(\theta_{n}=g_{i_{n}} \mid \theta_{1}=g_{i_{1}}, \cdots, \theta_{k}=g_{i_{k}}, \xi_{t}\right)=\frac{p_{j_{n}} \psi_{i_{1} \cdots i_{k} j_{n}}^{(t)}\left(\xi_{t} ; \delta^{(t)}\left(i_{1}, \cdots, i_{n}\right)\right)}{\sum_{l_{n}} p_{l_{n}} \bar{\psi}_{i_{1} \cdots i_{k} l_{n}}^{(t)}\left(\xi_{t} ; \delta^{(t)}\left(i_{1}, \cdots, i_{n}\right)\right)},
$$

where

$$
\begin{aligned}
& \phi_{i_{1} \cdots i_{k} l_{n}}^{(t)}\left(\xi_{t} ; \delta^{(t)}\left(i_{1}, \cdots, i_{n}\right)\right) \\
& =\sum_{j_{k+1}} \cdots \sum_{j_{n-1}} p_{j_{k+1}} \cdots p_{j_{n-1}} \phi_{i_{1} \cdots i_{k} j_{k-1} \cdots j_{n-1} l_{n}}^{(t)}\left(\xi_{t} ; \delta^{(t)}\left(i_{1}, \cdots, i_{n}\right)\right) .
\end{aligned}
$$

By the definition each player in $K$ obtains the same amount of information on $\theta_{n}$.

According to the theorems on conditional entropy we obtain

$$
I_{1}^{(t)}\left(\theta_{n} ; \delta(1), \cdots, \delta(n)\right) \leqq I_{K}^{(t)}\left(\theta_{n} ; \delta(1), \cdots, \delta(n)\right)
$$

for all $t=1,2, \cdots$. Thus the cooperation brings each member more amount of information than he gets by himself. We show a sufficient condition under which a player in $K$ can get the full information on $\theta_{n}$.

THEOREM 7. If for every $i_{1}, \cdots, i_{n}$ and $j_{n} \neq i_{n}$

$$
\lim _{t \rightarrow \infty} \int_{S^{t+1}}\left\{\left\{\psi_{i_{1} \cdots i_{k} i_{n}}^{(t)}\left(\xi_{t} ; \delta^{(t)}\left(i_{1}, \cdots, i_{n}\right)\right) \cdot \psi_{i_{1} \cdots i_{k} j_{n}}^{(t)}\left(\xi_{t} ; \delta^{(t)}\left(i_{1}, \cdots, i_{n}\right)\right)\right\}^{1 / 2} d \xi_{t}=0\right.
$$

then every player in $K$ obtains the full information on $\theta_{n}$.

PRoOF. By Lemma 1 there exists a constant $C>0$ such that

$$
\begin{aligned}
& E_{i_{1} \cdots i_{n}}\left[H\left(\theta_{n} \mid \theta_{1}, \cdots, \theta_{k}, \xi_{t}\right) \mid \theta_{1}=g_{i_{1}}, \cdots, \theta_{n}=g_{i_{n}}\right] \\
& \leqq C \int_{S^{t+1}} \sum_{j_{n} \neq i_{n}}\left\{\operatorname{Pr}\left(\theta_{n}=g_{j_{n}} \mid \theta_{1}=g_{i_{1}}, \cdots, \theta_{k}=g_{i_{k}}, \xi_{t}\right)\right\}^{1 / 2} \\
& \text { - } \phi_{i_{1} \cdots i_{n}}^{(t)}\left(\xi_{t} ; \delta^{(t)}\left(i_{1}, \cdots, i_{n}\right)\right) d \xi_{t} \\
& \leqq C \int_{S^{t+1}} \sum_{j_{n} \neq i_{n}}\left\{\frac{p_{j_{n}} \psi_{i_{1} \cdots i_{k} i_{n}}^{(t)}\left(\xi_{t} ; \delta^{(t)}\left(i_{1}, \cdots, i_{n}\right)\right)}{p_{i_{n}} \psi_{i_{1}(t) i_{k} j_{n}}^{(t)}\left(\xi_{t} ; \delta^{(t)}\left(i_{1}, \cdots, i_{n}\right)\right)}\right\}^{1 / 2} \\
& \cdot \phi_{i_{1} \cdots i_{n}}^{(t)}\left(\xi_{t} ; \delta^{(t)}\left(i_{1}, \cdots, i_{n}\right)\right) d \xi_{t} .
\end{aligned}
$$

Since

$$
p_{i_{k+1}} \cdots p_{i_{n-1}} \phi_{i_{1} \cdots i_{n}}\left(\xi_{t} ; \delta^{(t)}\left(i_{1}, \cdots, i_{n}\right)\right) \leqq \phi_{i_{1} \cdots i_{k} j_{n}}^{(t)}\left(\xi_{t} ; \delta^{(t)}\left(i_{1}, \cdots, i_{n}\right)\right),
$$

it follows

$$
\begin{aligned}
& E_{P} E_{\partial\left(i_{1}, \cdots, i_{n}\right)}\left[H\left(\theta_{n} \mid \theta_{1}, \cdots, \theta_{k}, \xi_{t}\right) \mid \theta_{1}=g_{i_{1}}, \cdots, \theta_{n}=g_{i_{n}}\right] \\
\leqq & C \int_{S^{t+1}} \sum_{j_{n} \neq i_{n}}\left\{\psi_{i_{1} \cdots i_{k} i_{n}}^{(t)}\left(\xi_{t} ; \delta^{(t)}\left(i_{1}, \cdots, i_{n}\right)\right) \psi_{i_{1} \cdots i_{k} j_{n}}^{(t)}\left(\xi_{t} ; \delta^{(t)}\left(i_{1}, \cdots, i_{n}\right)\right)\right\}^{1 / 2} d \xi_{t},
\end{aligned}
$$

which implies the theorem.

Considering Theorem 6 and Theorem 7, the inequality (15) implies (18). Thus taking account of the inequality of (17), we can say that the cooperation brings every member of the group much more benefits than he gets by himself. 


\section{Notes and remarks.}

We have defined optimal strategies with respect to the amount of ultimate information. However, in practical games another important problem is how soon one can get the maximum information on the opponents' power. Thus our next problems is to consider optimal strategies with respect to the time to get the maximum information. In the present game the information obtained at each time is not used to modify the strategies. In the next problem we also wish to consider such system that a player can employ the obtained information to determine his action at every time.

The information games can be treated from the viewpoint of learning theory. In the game player's object is to get information about another player, thus he learns some knowledge from the player. On the other hand a learning process of a teacher and a student can be considered as a kind of 2-person cooperative information game, where a optimal strategy of a teacher is a optimal reinforcement to teach the student. Therefore learning processes might be treated as information games.

\section{Acknowledgements.}

The auther would like to express his deep thanks to Professor Seigo Kanō of Kyushu University, who discussed and supported him throughout the work on information games. The author is also grateful to Hononary Professor Tosio Kitagawa, Professor Nagata Furukawa and the faculty of Research Institute of Fundamental Information Science of Kyushu University, who gave helpful suggestions.

\section{References}

[1] Feristen, A., Foundations of information theory, Mcgraw Hill, (1958).

[2] GuASU, S., Information theory with applications, Mcgraw Hill' (1977).

[3] KA.so, S. and KAI, Y., Information theoretical approaches in game theory, Bull. of Math. Stat., 19, No. 1-2, (1979), 37-43.

[4] KaI, Y. and Kanō, S., An information theory of game systems, Bull. of Math. Stat., 19, No. 3-4, (1981), 87-102.

[5] KARLin, S., Mathematical methods and theory in games, programming and economics, Addison-Wesley, (1959).

[6] Keneney, J.G., Snell, J.L. and KnApp, A.W., Denumerable Markov Chains, D. Van Nostrand, (1966).

[7] KoRsh, J.F., On decisions and information concerning an unknown parameter, Information and Control, 16, (1970), 123-127.

[8] Klllback, S., Information theory and statistics, Dover Publications, (1968).

[9] von Neumani, J, and Moagensteri, O., Theory of games and economic beoavior, Princeton Univercity, (1944).

[10] Neveu, J., Mathematical foundations of the calculus of probability, Holden-Day, (1965).

[11] RÉNYI, A., On the amount of information concerning an unknown parameter in a sequence of observations, Publ. of Math. Inst. of Hungarian Acad. Sci., 9, (1964), 617-624.

[12] RÉ.NYI, A,. On some basic problems of statistics from the point of view of information theory, Proceedings of 5-th Berkeley Symposium, 1, (1967), 531-543.

Communicated by S. Kanō 